\title{
Recover 3D Information of the Moving Object from Video Streams
}

\author{
Yu-tong Zheng ${ }^{1}$ and Ming Li and Fang Liao
}

Abstract Perception of the moving object in 3D from video streams has been one hot topic in computer vision. We present a fast method to reconstruct 3D information of the moving object from binocular video streams. System is assembled as two pipelines, technica are used to excavate the potential parallelism. With the corresponding points searching confined to very limited and credible region, the mismatching errors and time-consumed computation are reduced considerably. At the last, sparse depth map is calculated and then $3 \mathrm{D}$ contours and location of the object are estimated. The system is implemented and tested with outdoor and indoor moving object perception on $640 \times 480$ frame. Results show that the proposed method is improved in speed and stability. It can be used as a reference for autonomous navigation of mobile robot and object tracking.

Keywords Binocular, corresponding points, epipolar constraints, sparse depth map, autonomous navigation

\section{Introduction}

Making manmade machine capable to perceive the moving object in depth and co ntour from videos has received considerable attention in computer vision. Since th e projective transformation from 3D to image (2D) is intrinsic ambiguity, it is hard to recover $3 \mathrm{D}$ information from a single image [1]. We need more than one image to reconstruct $3 \mathrm{D}$, so correspondence points matching inevitably play an importan $t$ role in all the performance metrics. Along with achievement in intelligent compu ting, implementation technique and neurobiology relevant to vision $[2,3]$, many $\mathrm{n}$ ovel algorithms appear $[4,5,6,7]$, but further improvement is still wanted in univ ersal, stability, timeliness and accuracy. In many areas, such as human motion anal ysis, traffic monitoring, tracking, autonomous navigation, we want the system to work better like humans.

In this paper binocular are used to capture video streams synchronously, their two optical axis are parallel to each other and baseline between the two optical

\footnotetext{
${ }^{1}$ Yu-tong Zheng $(\bowtie)$

Information Engineering College, Minzu University of China, Beijing, 100081

e-mail: zhengyutong68@aliyun.com

Ming Li

Information Engineering College, Minzu University of China, Beijing, 100081

Fang Liao

Information Engineering College, Minzu University of China, Beijing, 100081
} 
centre hold invariant. When the moving object appears in the field, its 3D contour and location are reconstructed and updated in time. Section 2 describe the architecture of the whole system, section 3 describe the detail of the implementation, section 4 shows the development environment and tested results, and finally section 5 gives the conclusion.

\section{Design Scheme}

Simulating the human vision, the hardware of the system consists of two CMOS c ameras and one PC. Two cameras with identical parameters, fixed baseline and pa ralleled optical axis grab the same scene from two different positions synchronous ly; the left camera coordinate is regard as world coordinate. The intrinsic paramete rs and the poses of cameras are calibrated and rectified in advance precisely, so dis tortion are eliminated and calculation of subsequent process will reduce. The desig $\mathrm{n}$ scheme is shown in Fig.1. The whole system is assembled as two pipelines, whil e early in the program the information coming from the two cameras are processed in a parallel way.

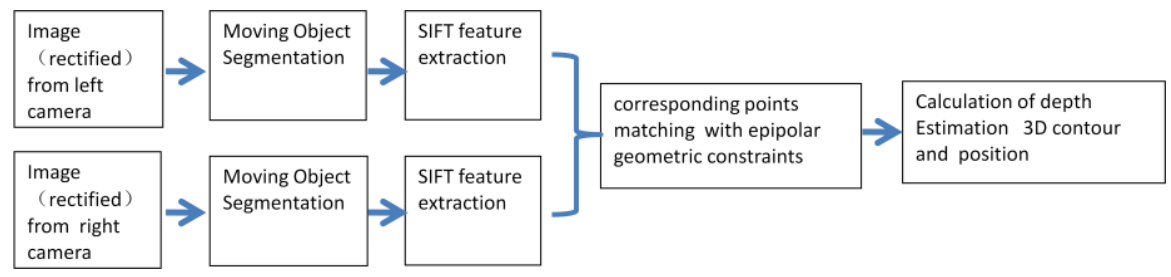

Fig.1 design scheme

Segmentation and the subsequent SIFT feature [8] extraction are processed syn chronously in two pipelines. The next step merged together is searching for corres ponding points under epipolar geometry constraints [1]. This step has key effect $\mathrm{o}$ $\mathrm{n}$ the ultimate real-time, accuracy and stability, we adopt an improved algorithm $\mathrm{w}$ hich will be described in detail .Triangulation is used for the calculation of depth $\mathrm{f}$ rom disparity. Along with the objects moving initiatively, the sparse depth maps ar e updated and show on the screen. Finally, the 3D outline of the moving object an $\mathrm{d}$ its location are estimated.

The test results show that the proposed method is reasonable improved in speed. The why behind are some tricks as follows: the first is two pipeline used in the early stage; the second is the limited and valid matching area; the third reason is the improved stereo correspondence algorithm. The test results also show that the improvement in robustness, the reason in nature comes from the advantages of SIFT itself, SIFT feature is invariant to image scaling, rotation, illumination, viewpoint and well localized in spatial, so it will reduce the probability of disruption by occlusion, clutter, or noise [8].If the relative position of binocular camera remains unchanged, the system can be used for perception of the moving object in $3 \mathrm{D}$ with no need for calibration and rectification in operative mode and can be expanded for multi-target tracking and 3D modeling in real-time. 


\section{The details of the implementation}

In this section, we describe the details step by step and the improved corresponding point match algorithm is proposed in the latter part.

\subsection{Calibration and Stereo-rectification}

Usually the lens has certain distortion. In many scenarios, eliminating the image di stortion is the chief problem of image pre-processing. By calibration[9,11], we can get the camera intrinsic parameters, including focal length, the optical centre, dist ortion coefficient and relative orientation of the cameras.Images can be undistorte $\mathrm{d}$ in a pre-processing step using distortion coefficients obtained during calibration.

Image stereo-rectification $[10,11]$ is the process by which two images of the $\mathrm{s}$ ame solid scene undergo homographic transforms, so that their corresponding epip olar lines coincide and become parallel to the $\mathrm{x}$-axis of image. So, given $\mathrm{x}$ in the le $\mathrm{ft}$ image from the pair of stereo-rectified images, the search domain for $\mathrm{x}$ ' in the ri ght image corresponding to $\mathrm{x}$ is restricted on the same horizontal scan line.

Bouquet's Algorithm [12] is exploited for rectifying calibrated cameras. In OP ENCV [13, 14], function void stereorectify ( ) computes rectification transforms $\mathrm{f}$ or each head of calibrated stereo camera.

\subsection{Moving Object Segmentation}

Experiment shows that when tracking objects moving very fast, the target missing will happen occasionally if algorithm such as Mean-shift and SAD template applie d. Segmentation [15] based on differential of sequential frame might have weakne ss such as slur and cavity, but missing target is not likely to happen. We exploit an improved method based on differential image of three consecutive frames. Details are as follows: first, two differential image according to the frame K-1 and the fol lowed frame $\mathrm{K}$, frame $\mathrm{K}$ and the followed $\mathrm{K}+1$ are acquired; second, spot with pix el value greater than the threshold is regarded as the target area, opposite as the ba ckground; finally intersection of the target areas is calculated, the result is accepte $\mathrm{d}$ as rough estimate of moving objects.

\subsection{Feature Extraction}

David Lowe put forward the SIFT features to describe image in 1989 [8]. It is a lo cal feature, invariant to image scaling, rotation and robust to illumination, viewpoi nt, occlusion, clutter, or noise. It has been validated in image retrieval, tracking, i mage fusion and many other applications. The reason why we select it is stability and straightforward use for disparity calculation. SIFT feature point $S \in \boldsymbol{\Re}^{133}$ is a high dimension data including scale, location, orientation, key point descriptor (12 8 dimension).By feature extraction two images are represented by two SIFT featur e vector respectively. 


\subsection{Corresponding Point Match with Epipolar Geometric Con- straints}

Corresponding point match is an important and difficult task, its aim is to find the corresponding point if the source image point has been given.

Many matching algorithms [16] have been explored these years which can be $\mathrm{d}$ ivided into two kinds: sparse matching and dense matching. Dense matching matc hes all the pixels in the image. Region around the pixel is selected, according to th e gray scale distribution or some other feature of the region, the comparability or $r$ elativity of the region in another image be obtained. This method has the problem of huge computation and mismatching errors. Usually texture images show a bette $r$ performance, but if the region is too large then blocking effect appears. The adva ntage is that dense depth map is straightforward. Sparse matching selects the stron $\mathrm{g}$ feature points for image matching, so feature extraction need be done first and th en decides matching point in a candidates queue. Sparse depth map is obtained dir ectly; the interpolation or affine transformation is required for dense depth map. B ut owing the blindness of the interpolation, it shows shortage in describe 3D struct ure in details. Due to no need to search in the whole image, the computation is red uced considerably. But the feature points are required to have definite characteristi cs in order to obtain high quality signals and stability.

In consideration of the needs of the autonomous navigation of moving robot, $\mathrm{w}$ e exploit a sparse matching algorithm using epipolar geometric constraints which i $\mathrm{s}$ the most fundamental and reliable geometric constraints in stereo vision. The pro jection $\mathrm{X}$ in the image plane of the point in the scene is on the corresponding epip olar line of the $X^{\prime}$ in another image plane [1]. If the parameters and the poses of ca meras are calibrated beforehand precisely and rectified to make the two image pla nes parallel, then baseline intersects the image plane at infinity, and Epipolar lines are parallel to $\mathrm{X}$ axis, so searching will be on the same horizontal level. The pseud o code of the algorithm is as follows:

Assume:

Input:

$$
\begin{aligned}
& L=\left\{V_{k} \mid V_{k} \in \text { FeaturePoint from Left Image }\right\} \\
& R=\left\{V_{k} \mid V_{k}^{\prime} \in \text { FeaturePoint from Right Image }\right\}
\end{aligned}
$$

Output:

$$
\text { Match }_{l, r}=\left\{\left\langle P_{l}, P_{r}\right\rangle \mid P_{l} \in V_{k}, P_{r} \in V_{k}^{\prime} \text { and } \in \text { corresponding points of } \mathrm{P}\right\}
$$

Step1: let

$$
\begin{aligned}
& L=\bigcup_{m=1}^{M}\left\{P_{m} \mid P_{m} \in \mathrm{V}_{\mathrm{k}} \text { have the identical Y coordinate }\right\} \\
& R=\bigcup_{m=1}^{M}\left\{P_{m} \mid P_{m}^{\prime} \in \mathrm{V}_{\mathrm{k}}^{\prime} \text { have the identical Y coordinate }\right\} \\
& M \in 1,2,3 \ldots \ldots Y_{\max }
\end{aligned}
$$

And denote subsets as: 


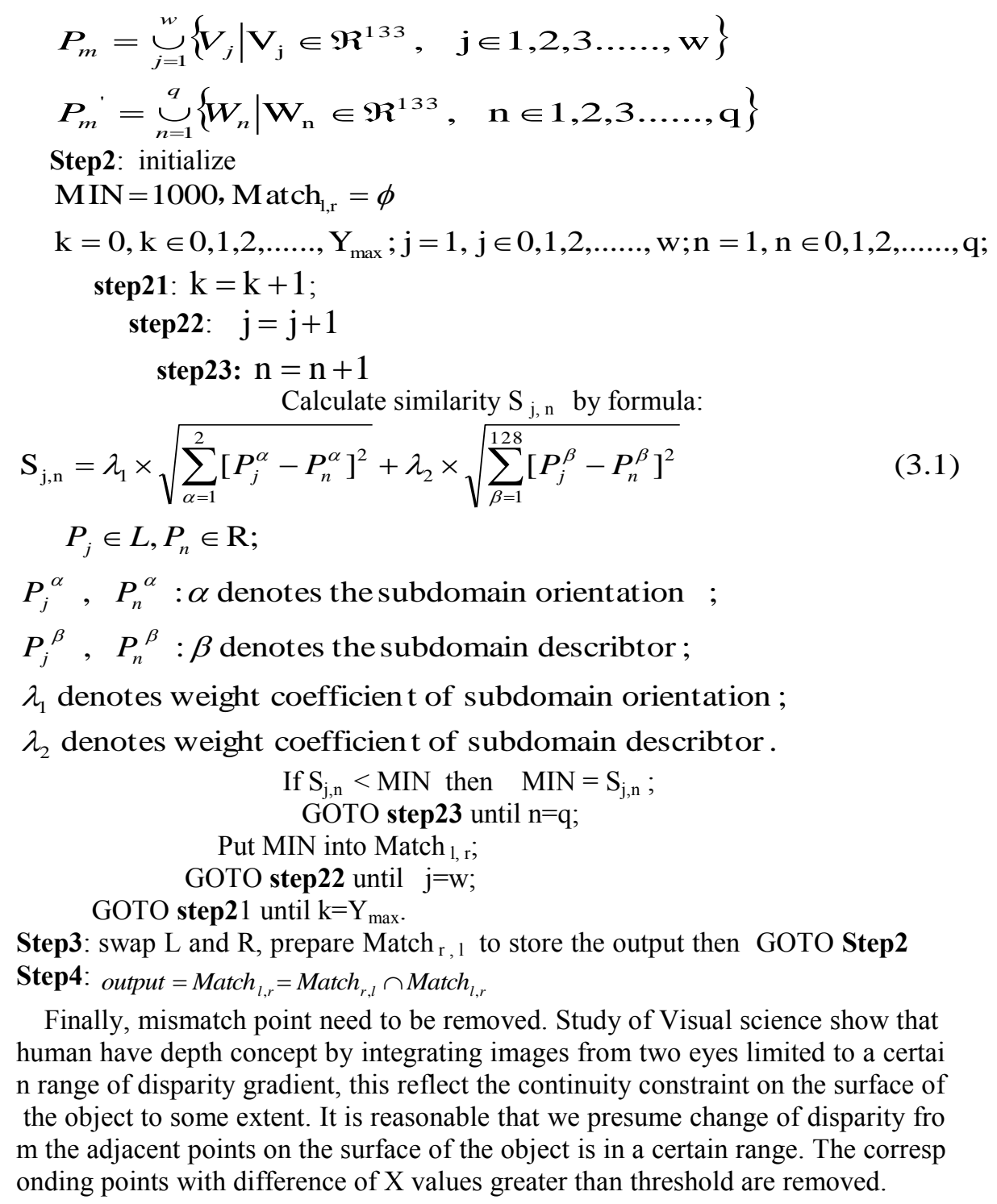

\subsection{Calculation of depth and estimation 3D contours and position}

Feature point $\mathrm{P} \quad(\mathrm{X}, \mathrm{Y}, \mathrm{Z})$ in the scene is projected on the left image plane as $\mathrm{P}_{1}$ $\left(\mathrm{x}_{1}, \mathrm{y}_{1}\right)$ and right as $\operatorname{Pr}\left(\mathrm{x}_{\mathrm{r}}, \mathrm{y}_{\mathrm{r}}\right), 3 \mathrm{D}$ information in the world coordinate can be calculated based on triangulation. Formulas are: 


$$
\begin{aligned}
& Z=\frac{f B}{\left|x_{l}-x_{r}\right|} \\
& X=\frac{B x_{l}}{\left|x_{l}-x_{r}\right|} \\
& Y=\frac{B y_{l}}{\left|x_{l}-x_{r}\right|}
\end{aligned}
$$

B denotes the distance between the optic centers; it is $150 \mathrm{~mm}$ in this system, $\mathrm{f}$ denote camera focal length and it is 820 pixels. Sparse depth map can be obtaine $\mathrm{d}$ directly from sparse matching. Fig.2 is sparse depth map of the object moving pa rallel to the camera plane, horizontal ordinate is frame, the unit is frame, vertical c oordinate is the depth, unit is millimeters, for convenience of observation, and unit in the figure is set to the decimeter. Fig. 3 is depth map of the object moving relati ve vertical to the camera plane.

Fig.4 shows the 3D of contour and location of the moving object, respectively $22,36,42$ frames, the unit is millimeters. For facilitate observation, unit in the fig ure is set to the decimeter.

In some application such as $3 \mathrm{D}$ reconstruction where detail information about $\mathrm{t}$ he structure of the object surface is needful, interpolation or affine transformation will be exploited to calculate dense depth map. In the application such as autono mous navigation of mobile robot, extreme are used to estimate the external contou r. We use the arithmetic mean $P_{i} \quad(X, Y, Z)$ (subscript i denote all the feature poin ts) estimate object's center of mass $P_{c}$. The estimate of the contour $\Lambda$ is a cuboids, the formula is as follows:

$$
\Lambda=\Omega_{1} \cap \Omega_{2} \cap \Omega_{3} \cap \Omega_{4} \cap \Omega_{5} \cap \Omega_{6}
$$

$\Omega_{1}$ and $\Omega_{2}$ are two planes parallel to the camera plane XY, the distance from the $\mathrm{XY}$ are $Z_{\min }$ and $Z_{\max } ; \Omega_{3}$ and $\Omega_{4}$ are two planes parallel to the plane $\mathrm{XZ}$, the distance from the optic center of the left the camera are $\mathrm{Y}_{\min }$ and $\mathrm{Y}_{\max } ; \Omega_{5}$ and $\Omega_{6}$ are two planes parallel to the plane $\mathrm{YZ}$, the distance from the optic center of the left the camera are $\mathrm{X}_{\text {min }}$ and $\mathrm{X}_{\max }$. 


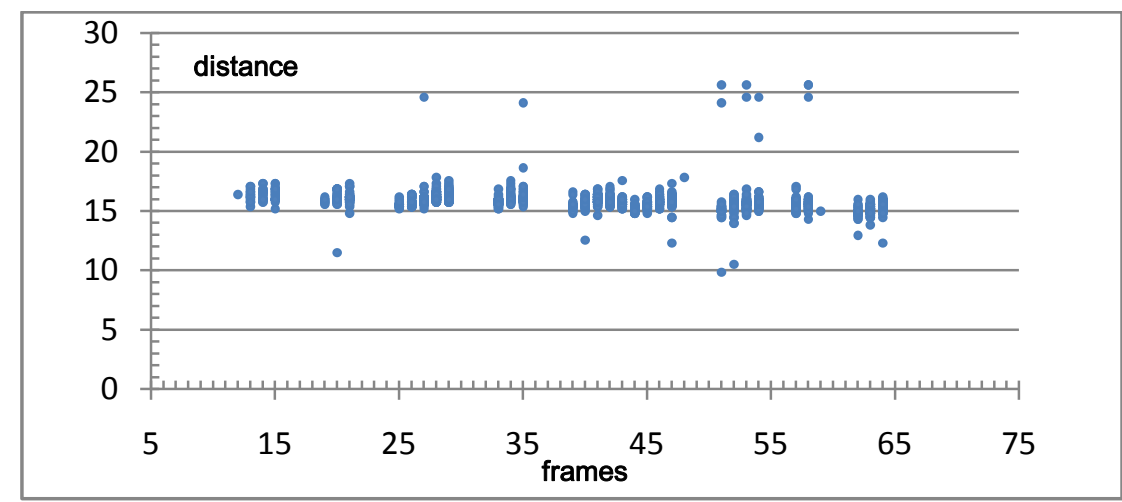

Fig. 2 sparse depth map of the object moving parallel to the camera plane

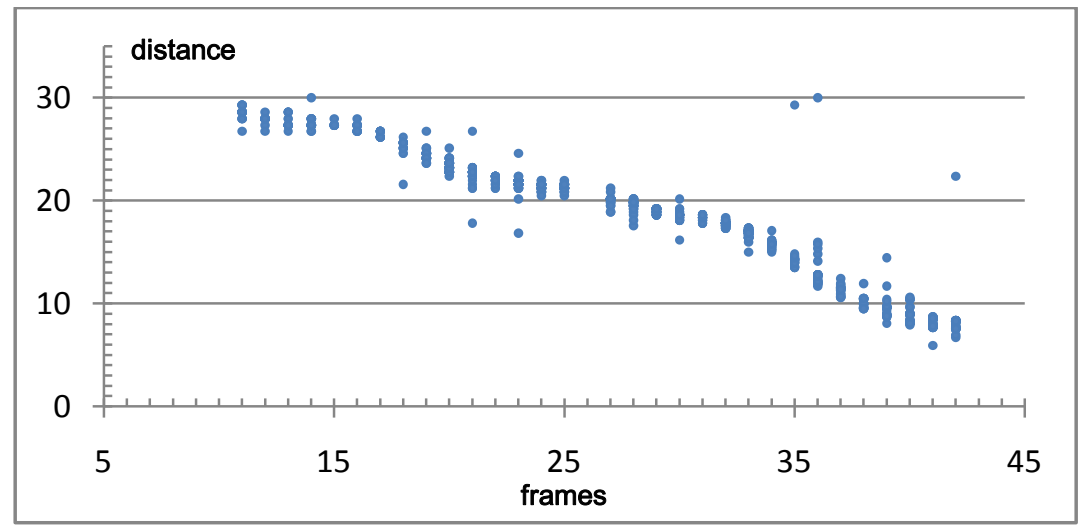

Fig. 3 sparse depth map of the object moving vertical to the camera plane

\section{Results}

We achieved a binocular system built on PC platform (Core i3-2120 3.30GHz, RA $\mathrm{M} 2 \mathrm{G})+$ two CMOS cameras in the form of two pipeline, it can be used for recov er 3D information of the moving object from video stream. The programming envi ronment is OPENCV+VISUAL STUDIO 2008, programming language is C and C ++ . The system has a satisfied speed and stability, where continuous image output without obvious dithering phenomenon. Fig. 4 shows that the shape composed by feature points in each frame remains generally stable, therefore the steady distribut ion of SIFT points is a valuable suggestion deserved further excavation for reconst ructing the object $3 \mathrm{D}$ outline. 


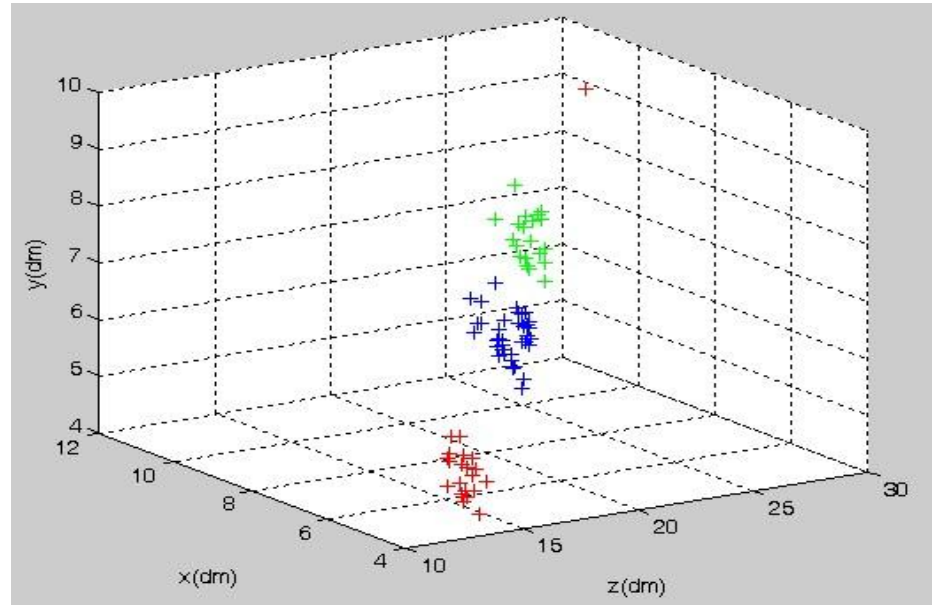

Fig. 4 3D display of contour and location of the moving object

\section{Conclusions and Future Work}

With the PC platform, the system receives data from outside two CMOS digital ca meras, reconstructs sparse 3D image of the moving object with two peculiar pipeli nes, shows the result on VGA monitor in time. Owing to its good performance in $r$ eal-time and robustness, it can be used as a reference for autonomous navigation o f mobile robot and object tracking.

The main feature of the system is as follows:

Multi-pipeline and Multi-threading technology improve the system parallelism to the greatest extent; Frame differential method is used to estimate the moving o bject, it improves the speed of the subsequent stages by reducing calculation limite $\mathrm{d}$ to a particular area; Improved stereo correspondence algorithm greatly reduces $t$ he search range. All these measures make the whole task showed a good performa nce in real-time.

SIFT features show robustness and powerful resistance to noise in many applic ation, it is a guarantee for the correctness of the corresponding match.

In the case of multi-targets, the targets can still be estimated by segmentation a nd Multi-pipeline and Multi-threading technology still works, so the system can be adjusted to multi- target tracking conveniently.

There is no need for calibration and rectification in operative mode. If the relat ive position of binocular camera remains unchanged, the system still works even if the binocular cameras are in motion.

Time analysis shows that feature extraction is bottleneck in pipeline. If we do s omething such as substitute SURF for SIFT to optimize, we can further improve th e real-time performance of the system. 


\section{References}

1. R. Hartley and A. Zisserman. (2005). Multiple View Geometry in Computer Vision, $2^{\text {nd }}$ ed.. Cambridge University Press, Cambridge

2. D. Leopold and N. K. Logothetis. (1996).Activity changes in early visual cortex reflect monkeys' precepts during binocular rivalry. Nature:379, 549-553

3. Takanori Uka and Gregory C. DeAngelis. (2006). Linking Neural Representation to Function in Stereoscopic Depth Perception: Roles of the Middle Temporal Area in coarse versus Fine Disparity Discrimination. The Journal of Neuroscience, June 21, 26(25): 6791-6802

4. Guan, S., Klette, R. (2006).Belief Propagation on edge image for stereo analysis of image sequences. In Proceedings Robot Vision. LNCS 4931, p. 291 - 302.

5. Y Boykov, O Veksler, R Zabih, Fast approximate energy minimization via graph cuts, IEEE Trans PAMI, 2001,23(11): 1222-1239

6. D. Scharstein and R. Szeliski. (2002). Taxonomy and evaluation of dense two-frame stereo correspondence algorithms.International Journal of Computer Vision, April-June $47(1 / 2 / 3): 7-42$

7. Guo Longyuan,Xia Yongquan,Yang Jingyu.(2008).Adaptive Search Region Fast Area_Based Stereo Correspondence.IEEE Trans.Image and Signal Processing,2:27-30

8. Lowe D G. (2004).Distinctive image features from scale-invariant key points IJCV, 60(2): $91-110$

9. J.-Y.Bouguet.(2001).Camera calibration toolbox for Matlab, http://www.vision.caltech.edu/ bouquet/calib\_doc/

10. Luca Lucchese.(2005).Geometric calibration of digital cameras through multi-view rectification, Image and Vision Computing ,23,517-53

11. Shimizu, M., Okutomi, M. (2008).Calibration and rectification for reflection stereo. In IEEE Conference on Computer Vision and Pattern Recognition CVPR. Anchorage (USA), p. $1-8$.

12. http://www.vision.caltech.edu/bouguetj/calib doc/index.html. 2004.

13. http://sourceforge.net/projects/opencvlibrary/

14. G. Bradsky and A. Kaehler. (2008).Learning OpenCV: Computer Vision with the OpenCV library. O'Reilly, Sebastopol, CA

15. Lukac, P., Hudec, R., Benco, M., Kamencay, P., Dubcova, Z., Zachariasova, M. (2011).Simple comparison of image segmentation algorithms based on evaluation criterion. In Proceedings of $21^{\text {st }}$ International Conference Radio electronics. Brno (Czech Republic), p. $233-236$.

16. Kuhl, A. (2005).Comparison of stereo matching algorithms for mobile robots. Centre for Intelligent Information Processing System. University of Western Australia, p. 4-24. 